\title{
Efficacy of umeclidinium/vilanterol versus umeclidinium and salmeterol monotherapies in symptomatic patients with COPD not receiving inhaled corticosteroids: the EMAX randomised trial
}

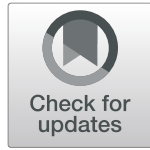

François Maltais ${ }^{1 *}$, Leif Bjermer ${ }^{2}$, Edward M. Kerwin ${ }^{3}$, Paul W. Jones ${ }^{4}$, Michael L. Watkins ${ }^{5}$, Lee Tombs ${ }^{6}$, lan P. Naya ${ }^{4}$, Isabelle H. Boucot ${ }^{4}$, David A. Lipson ${ }^{7,8}$, Chris Compton ${ }^{4}$, Mitra Vahdati-Bolouri ${ }^{9}$ and Claus F. Vogelmeier ${ }^{10}$

\begin{abstract}
Background: Prospective evidence is lacking regarding incremental benefits of long-acting dual- versus monobronchodilation in improving symptoms and preventing short-term disease worsening/treatment failure in low exacerbation risk patients with chronic obstructive pulmonary disease (COPD) not receiving inhaled corticosteroids.

Methods: The 24-week, double-blind, double-dummy, parallel-group Early MAXimisation of bronchodilation for improving COPD stability (EMAX) trial randomised patients at low exacerbation risk not receiving inhaled corticosteroids, to umeclidinium/vilanterol $62.5 / 25 \mu \mathrm{g}$ once-daily, umeclidinium $62.5 \mu \mathrm{g}$ once-daily or salmeterol $50 \mu \mathrm{g}$ twice-daily. The primary endpoint was trough forced expiratory volume in $1 \mathrm{~s}\left(\mathrm{FEV}_{1}\right)$ at Week 24. The study was also powered for the secondary endpoint of Transition Dyspnoea Index at Week 24. Other efficacy assessments included spirometry, symptoms, heath status and short-term disease worsening measured by the composite endpoint of clinically important deterioration using three definitions.
\end{abstract}

Results: Change from baseline in trough $\mathrm{FEV}_{1}$ at Week 24 was $66 \mathrm{~mL}$ (95\% confidence interval [Cl]: 43, 89) and $141 \mathrm{~mL}$ $(95 \%$ Cl: 118,164$)$ greater with umeclidinium/vilanterol versus umeclidinium and salmeterol, respectively (both $p<0.001)$. Umeclidinium/vilanterol demonstrated consistent improvements in Transition Dyspnoea Index versus both monotherapies at Week 24 (vs umeclidinium: 0.37 [95\% Cl: 0.06, 0.68], $p=0.018$; vs salmeterol: 0.45 [95\% Cl: 0.15, 0.76], $p=0.004)$ and all other symptom measures at all time points. Regardless of the clinically important deterioration definition considered, umeclidinium/vilanterol significantly reduced the risk of a first clinically important deterioration compared with umeclidinium (by $16-25 \%[p<0.01]$ ) and salmeterol (by $26-41 \%[p<0.001]$ ). Safety profiles were similar between treatments.

Conclusions: Umeclidinium/vilanterol consistently provides early and sustained improvements in lung function and symptoms and reduces the risk of deterioration/treatment failure versus umeclidinium or salmeterol in symptomatic patients with low exacerbation risk not receiving inhaled corticosteroids. These findings suggest a potential for early use of dual bronchodilators to help optimise therapy in this patient group.

Keywords: COPD, Lung function, Dyspnoea, Clinically important deterioration, Bronchodilator therapy

\footnotetext{
* Correspondence: Francois.Maltais@fmed.ulaval.ca

${ }^{1}$ Centre de Pneumologie, Institut universitaire de cardiologie et de

pneumologie de Québec, Université Laval, Québec, Canada

Full list of author information is available at the end of the article
}

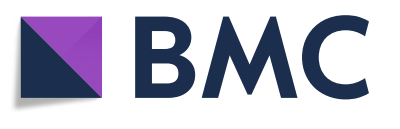

(c) The Author(s). 2019 Open Access This article is distributed under the terms of the Creative Commons Attribution 4.0 International License (http://creativecommons.org/licenses/by/4.0/), which permits unrestricted use, distribution, and reproduction in any medium, provided you give appropriate credit to the original author(s) and the source, provide a link to the Creative Commons license, and indicate if changes were made. The Creative Commons Public Domain Dedication waiver (http://creativecommons.org/publicdomain/zero/1.0/) applies to the data made available in this article, unless otherwise stated. 


\section{Background}

Long-acting bronchodilators, including long-acting muscarinic antagonists (LAMAs) and long-acting $\beta_{2}$-agonists (LABAs), form the basis of maintenance therapy in chronic obstructive pulmonary disease (COPD) [1]. The recommended first-line treatment for patients with symptomatic COPD at low exacerbation risk is LAMA or LABA monotherapy. LAMA/LABA dual therapy is also considered appropriate initial therapy in patients who experience severe breathlessness; however, based on current evidence, a stepwise approach is generally preferred [1].

LAMA/LABA therapy is more effective than LAMA or LABA monotherapy for improving lung function in patients with COPD; however, variability exists across studies in the reported magnitude of improvements of symptoms and health status with dual bronchodilation [2-8]. Until now, trials comparing dual- versus mono-bronchodilator therapy have generally included large proportions of patients with predominantly low exacerbation risk, but who were nevertheless using concurrent inhaled corticosteroids (ICS) $[2,5,9,10]$. Concurrent use or recent withdrawal of ICS may limit the generalisability of the results of such bronchodilator studies and confound the results regarding the incremental benefits of LAMA/LABAs compared with mono-bronchodilator therapies $[11,12]$. A recent integrated analysis of 23 randomised controlled trials (RCTs; $n=23,213$ ) evaluating the effect of LAMA/LABAs versus LAMAs, LABAs or placebo, on lung function, symptoms and exacerbation rates reported that $54 \%$ of enrolled patients were using concurrent ICS [4]. In the only 24-week Phase III RCT comparing the LAMA/LABA umeclidinium/vilanterol (UMEC/VI) versus UMEC and VI monotherapy, half of the patients continued using concurrent ICS [7]. That study demonstrated improvements in lung function and rescue medication use with UMEC/VI versus both monotherapies but did not demonstrate any incremental improvements in self-reported breathlessness or other patient-reported outcomes (PROs) [7]. Patients not receiving ICS treatment are an important and prevalent category of patients and usually reflect earlier stages of COPD. Consequently, further trials of UMEC/VI versus mono-bronchodilator therapy in symptomatic patients not receiving concurrent ICS are warranted to prospectively assess treatment optimisation in this patient population.

A composite endpoint of clinically important deterioration (CID) was recently developed to assess short-term disease worsening across multiple dimensions. The CID assesses deterioration from baseline in individual patients in terms of forced expiratory volume in $1 \mathrm{~s}\left(\mathrm{FEV}_{1}\right)$, and/or a $\mathrm{PRO}$, and/or the occurrence of a moderate or severe COPD exacerbation $[8,13-16]$. Assessing both symptom improvement and risk of short-term disease deterioration (i.e. treatment failure) is important to fully quantify the adequacy of maintenance therapy in individual patients, as since many symptomatic patients fail to achieve clinically relevant improvements or achieve relative disease stability, and instead experience deterioration of their disease [14]. To date, CID has only been evaluated retrospectively in the comparison of dual-versus mono-bronchodilators [8, 13-16].

To better understand the role of dual bronchodilation in symptomatic low exacerbation risk patients, we conducted the large prospective trial, Early MAXimisation of bronchodilation for improving COPD stability (EMAX), which monitored improvements in spirometry, a range of PROs and CID, with three different bronchodilators: UMEC/VI, UMEC and salmeterol.

\section{Methods}

\section{Study design}

This 24-week, multicentre, randomised, double-blind, double-dummy, 3-arm, parallel-group trial (NCT03034915; GSK study: 201749) was conducted between June 2017 and June 2018 in 213 centres in Germany, USA, Argentina, Sweden, Canada, Italy, South Africa, Netherlands, Spain, Australia, France, and Mexico. Patients were randomised 1: 1:1 to once-daily fixed-dose combination UMEC/VI (62.5/ $25 \mu \mathrm{g}$ ) via the ELLIPTA inhaler and twice-daily placebo via the DISKUS inhaler, once-daily UMEC $(62.5 \mu \mathrm{g})$ via ELLIPTA inhaler and twice-daily placebo via DISKUS, or twice-daily salmeterol $(50 \mu \mathrm{g})$ via DISKUS and once-daily placebo via ELLIPTA inhaler (Additional file 6: Figure S1). Salmeterol was selected as a comparator as no once-daily LABAs were approved at standard doses in all countries participating in the study; its use also allowed the LABA treatment to be easily blinded compared with the alternative twice-daily LABA, formoterol. UMEC was selected as it is a component of the dual bronchodilator and it has also demonstrated superior lung function benefits compared with tiotropium [17].

This study was performed according to the Declaration of Helsinki and received appropriate ethical approval. All patients provided written informed consent via a form signed at either the Pre-screening or Screening visit.

\section{Patients}

Eligible patients were $\geq 40$ years of age, current/former smokers ( $\geq 10$ pack-years smoking history), with a COPD diagnosis (American Thoracic Society/European Respiratory Society definition), pre- and post-salbutamol $\mathrm{FEV}_{1} /$ forced vital capacity (FVC) ratio $<0.7$, post-salbutamol $\mathrm{FEV}_{1}$ of $\geq 30-\leq 80 \%$ predicted (Global Initiative for Chronic Obstructive Lung Disease [GOLD] stage 2/3), COPD Assessment Test (CAT) score $\geq 10$, with $\leq 1$ moderate exacerbation and no severe exacerbations in the previous year. Before screening and during the 4-week run-in period, bronchodilator maintenance therapy was limited to a LAMA or LABA. All patients were required 
to be ICS and ICS/LABA free for $\geq 6$ weeks and LAMA/ LABA free for $\geq 2$ weeks prior to run-in. As-needed salbutamol was allowed throughout all study phases.

\section{Randomisation and masking}

Eligible patients were stratified by country and longacting bronchodilator use during run-in and were centrally randomised within each country using an interactive web response system (Registration and Medication Ordering System NG). All treatments and/or matched placebo in this double-blind, double-dummy trial were delivered via identical inhalation devices so that treatment was masked to patients, investigators and data analysts.

\section{Procedures}

Clinic visits occurred at Pre-screening/Screening, Randomisation (Day 1), and after 4, 12, and 24 weeks of treatment (Additional file 6: Figure S1). At the Pre-screening visit, demographic and concomitant medication information were collected, and COPD exacerbation history was assessed. Assessment of symptom-reported outcomes at clinic visits were conducted in the following order and before other study assessments: self-administered computerised-Baseline Dyspnoea Index (SAC-BDI; at randomisation visit), selfadministered computerised-Transition Dyspnoea Index (SAC-TDI; post randomisation), and global assessment of disease severity. Health status was assessed at clinic visits after the symptom-reported outcomes using St George's Respiratory Questionnaire (SGRQ), and CAT. Daily symptoms were evaluated using rescue salbutamol use and the Evaluating Respiratory Symptoms-COPD (E-RS), which were both captured via an electronic diary (e-diary) (Additional file 1: Table S1). Exacerbations were recorded by the physician at the Pre-screening visit and at every subsequent visit until completion of follow-up contact. Exacerbations requiring treatment with oral corticosteroids and/or antibiotics were categorised as moderate, and those requiring hospitalisation or an emergency room visit as severe. CID was prospectively analysed as a composite endpoint of time to first deterioration from baseline in trough $\mathrm{FEV}_{1}$, SGRQ total score, CAT score, and SAC-TDI focal score in addition to the occurrence of a moderate/severe exacerbation.

\section{Outcomes}

The primary endpoint was change from baseline in trough $\mathrm{FEV}_{1}$ at Week 24. Trough $\mathrm{FEV}_{1}$ at Week 24 was defined as the mean of the $\mathrm{FEV}_{1}$ values obtained 23 and $24 \mathrm{~h}$ after dosing on the previous day (Day 167) as recorded in the e-diary. Additional spirometry assessments included trough $\mathrm{FEV}_{1}, \mathrm{FVC}$, and inspiratory capacity (IC) over 24 weeks. Patient-reported symptom-based assessments included SAC-TDI for breathlessness, global assessment of disease severity, daily rescue salbutamol use, and E-RS respiratory symptoms total score. Health status assessments included SGRQ total score and CAT score. Response rates in individual patients were defined as $\geq 1$-unit improvement from baseline in SAC-TDI score [18], $\geq 2$-point reduction from baseline in E-RS total score [19], $\geq 4$-point reduction from baseline in SGRQ total score [20], and $\geq 2$-unit improvement from baseline in CAT score [21].

Time to first moderate or severe exacerbation was also assessed. Risk of a first CID was assessed in individual patients according to three composite definitions: A) a first moderate or severe exacerbation, and/or a trough $\mathrm{FEV}_{1}$ decrease from baseline of $\geq 100 \mathrm{~mL}$, and/or a deterioration in health status using SGRQ ( $\geq 4$ units from baseline); B) as per the first definition with a CAT deterioration $(\geq 2$ units from baseline) replacing a SGRQ deterioration; C) a $\mathrm{FEV}_{1}$-free CID definition including a first moderate or severe exacerbation, and/or a SGRQ deterioration, and/or a CAT deterioration, and/or a TDI deterioration ( $\geq 1$ unit decrease from baseline). Safety outcomes included incidence of adverse events (AEs) and serious AEs (SAEs).

\section{Statistical analysis}

This study was powered to detect differences in the primary endpoint and in SAC-TDI at Week 24. The primary treatment comparison for the primary endpoint was UMEC/VI versus UMEC. If the primary comparison was significant, this allowed inference of other treatment comparisons. The sample size calculation used a two-sided 5\% significance level and an estimate of between patient standard deviation (SD) for TDI of 2.94 units [7]. Based on these data, 727 evaluable patients per treatment arm were required to provide $90 \%$ power to detect a statistically significant difference in TDI if the true difference was 0.5 units (half the minimal clinically important difference) [18], between UMEC/VI and UMEC. With this number of evaluable patients per arm, the study would have $>99 \%$ power assuming a true treatment difference of $80 \mathrm{~mL}$ between UMEC/VI and UMEC for trough $\mathrm{FEV}_{1}$ at 24 weeks at the twosided 5\% significance level. This calculation used a trough $\mathrm{FEV}_{1} \mathrm{SD}$ of $240 \mathrm{~mL}$, based on prior results for trials comparing dual- versus mono-bronchodilators [2, 7]. The intent-to-treat (ITT) population included all randomised patients who received $\geq 1$ dose of study treatment. The primary analysis was a mixed model repeated measures analysis based on a two-sided hypothesis testing approach. Least squares (LS) mean and LS mean change from baseline analyses with estimated treatment differences and 95\% confidence intervals (CIs) are presented. Responder analyses with corresponding odds ratios (OR) and 95\% CIs were performed using a generalised linear mixed model. 
Time to first exacerbation hazard ratios (HR) and 95\% CIs were based on Cox proportional hazards model with covariates of treatment, stratum (number of bronchodilators per day during run-in), and geographical region. Time to first CID HRs and 95\% CIs were based on a Cox proportional hazards model with covariates of treatment, stratum (number of bronchodilators per day during run-in), geographical region, trough $\mathrm{FEV}_{1}$ at baseline, and SGRQ score at baseline. Safety endpoints were analysed descriptively. All analyses presented were pre-planned with the exception of time to study treatment withdrawal, which was post hoc.

This study was funded by GlaxoSmithKline (GSK study number: 201749 [NCT03034915]). GSK-affiliated authors had a role in study design, data analysis, data interpretation, and writing of the report and GSK funded the article processing charges and open access fee.

\section{Results}

Of 3828 patients screened, 2431 were randomly assigned to treatment; 6 patients did not receive study treatment and were excluded from the ITT population (Fig. 1). The most frequent reason for screening failure was not meeting the COPD severity inclusion criteria.

The ITT population comprised 2425 patients across the UMEC/VI $(n=812)$, UMEC $(n=804)$, and salmeterol $(n=809)$ treatment arms (Fig. 1). Overall, 2050/2425 (85\%) patients completed the study treatment period. Completion rates were highest for UMEC/VI (717/812 [88\%]) compared with UMEC (650/804 [81\%]) and salmeterol $(683 / 809$ [84\%]) with fewer patients in the UMEC/VI group withdrawing consent and withdrawing from the study due to lack of efficacy and other protocol-specified withdrawals (Fig. 1 and Additional file 7: Figure S2). A post hoc assessment demonstrated a decreased risk of

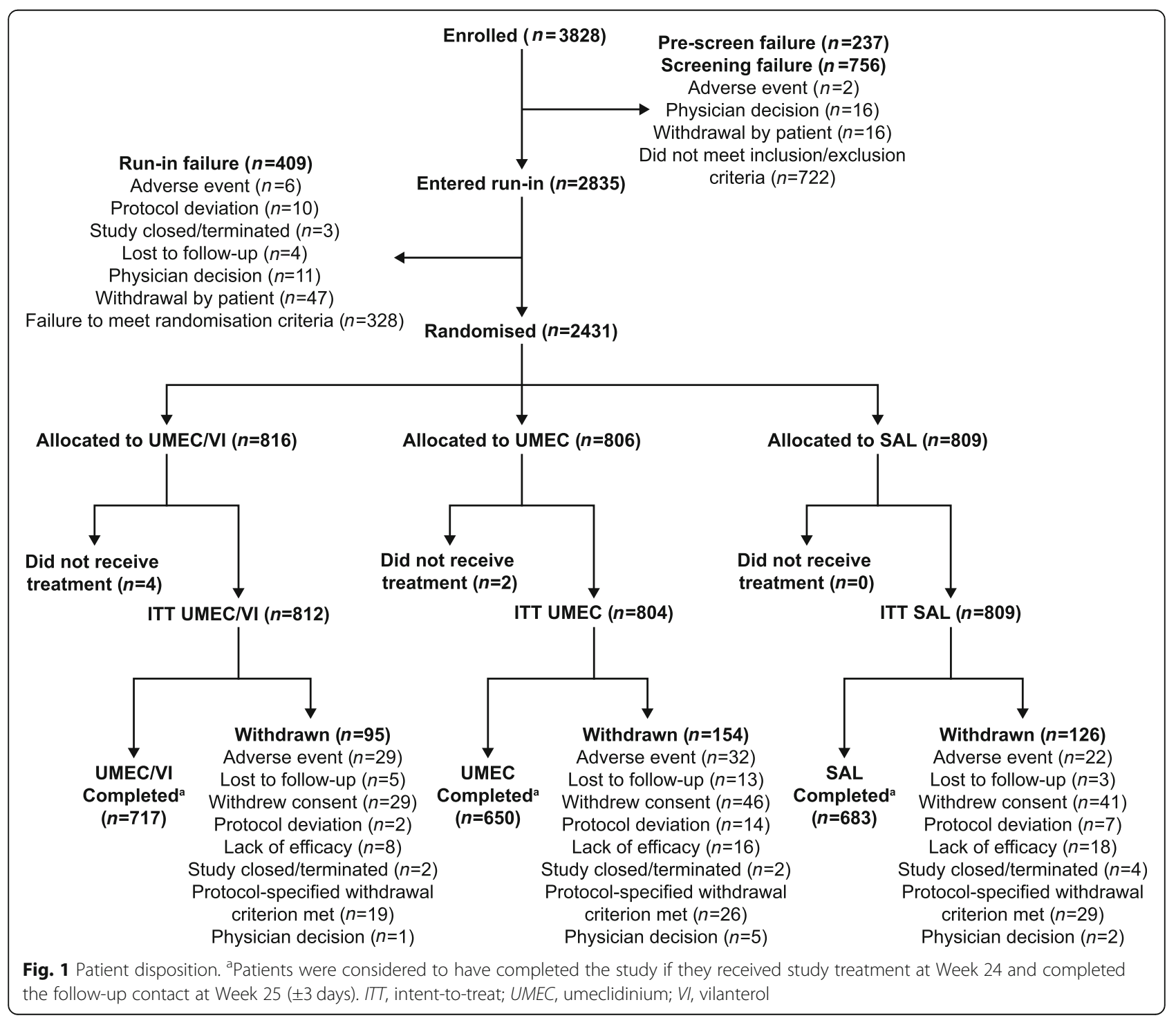


premature study withdrawal with UMEC/VI compared with UMEC (HR [95\% CI]: $0.60[0.46,0.77] ; p<0.001)$ and salmeterol (HR [95\% CI]: 0.73 [0.56, 0.96]; $p=0.022$ ).

Patient demographics and baseline characteristics were similar between treatment arms (Table 1); mean age was 64.6 years, 988/2425 (41\%) were female, and 1203/2424 $(50 \%)$ were current smokers. Mean post-salbutamol percent predicted $\mathrm{FEV}_{1}$ was $55.4 \%$ and mean CAT score at baseline was 19.2. Overall, 393/2425 (16\%) patients had one moderate exacerbation in the previous year.

The LS mean change from baseline in trough $\mathrm{FEV}_{1}$ at Week 24 was $122 \mathrm{~mL}$ for UMEC/VI, $56 \mathrm{~mL}$ for UMEC, and $-19 \mathrm{~mL}$ for salmeterol (Fig. 2). For the primary endpoint, change from baseline in trough $\mathrm{FEV}_{1}$ at Week

Table 1 Patient demographics and baseline characteristics

\begin{tabular}{|c|c|c|c|c|}
\hline Characteristic & UMECNI $(N=812)$ & $\operatorname{UMEC}(N=804)$ & $\mathrm{SAL}(N=809)$ & Total $(N=2425)$ \\
\hline Age, years, mean (SD) & $64.6(8.4)$ & $64.9(8.5)$ & $64.4(8.5)$ & $64.6(8.5)$ \\
\hline Female, n (\%) & 319 (39) & $327(41)$ & $342(42)$ & $988(41)$ \\
\hline \multicolumn{5}{|l|}{ Race, n (\%) } \\
\hline White & 767 (94) & $764(95)$ & 766 (95) & $2297(95)$ \\
\hline Black/African American & $24(3)$ & $23(3)$ & $25(3)$ & $72(3)$ \\
\hline American Indian/Alaska Native & $13(2)$ & $12(1)$ & $12(1)$ & $37(2)$ \\
\hline Asian & $5(<1)$ & $1(<1)$ & $1(<1)$ & $7(<1)$ \\
\hline Multiple ${ }^{a}$ & $3(1)$ & $4(<1)$ & $5(<1)$ & $12(<1)$ \\
\hline Current smoker at screening, n (\%) & $394(49)$ & $396(49)$ & $413(51)$ & $1203(50)$ \\
\hline Smoking pack-years, mean (SD) & $49.4(27.7)$ & $47.6(25.9)$ & $48.1(25.8)$ & $48.4(26.5)$ \\
\hline Use of $L A B D$ during run-in, $n(\%)^{b}$ & $531(65)$ & $521(65)$ & $524(65)$ & $1576(65)$ \\
\hline LAMA & $399(49)$ & $392(49)$ & $403(50)$ & $1194(49)$ \\
\hline LABA & $130(16)$ & $142(18)$ & $132(16)$ & $404(17)$ \\
\hline No maintenance medication during run-in, n (\%) & $250(31)$ & $250(31)$ & $249(31)$ & $749(31)$ \\
\hline Moderate COPD exacerbation history in prior year ${ }^{c}, \mathrm{n}(\%)$ & $123(15)$ & $124(15)$ & $146(18)$ & $393(16)$ \\
\hline Duration of COPD, years, mean (SD) & $8.8(6.9)$ & $7.8(6.0)$ & $8.3(6.7)$ & $8.3(6.6)$ \\
\hline Post-salbutamol FEV $1, \mathrm{~mL}$, mean (SD) & $1577(506)$ & $1609(503)$ & $1600(523)$ & $1595(511)$ \\
\hline Post-salbutamol \% predicted $\mathrm{FEV}_{1}$, mean (SD) & $54.9(12.8)$ & $55.9(12.6)$ & $55.6(12.8)$ & $55.4(12.7)$ \\
\hline Post-salbutamol FEV $/$ FVC, mean (SD) & $0.51(0.10)$ & $0.52(0.10)$ & $0.52(0.10)$ & $0.52(0.10)$ \\
\hline$\%$ reversibility to salbutamol, mean (SD) & $10.4(12.8)$ & $10.2(13.3)$ & $10.7(13.3)$ & $10.5(13.1)$ \\
\hline \multicolumn{5}{|l|}{ GOLD spirometric grade ${ }^{\mathrm{d}}, \mathrm{n}(\%)$} \\
\hline 2 & $518(64)$ & $529(66)$ & $522(65)$ & $1569(65)$ \\
\hline 3 & $294(36)$ & $271(34)$ & $286(35)$ & $851(35)$ \\
\hline Baseline $\mathrm{FEV}_{1}, \mathrm{~mL}$, mean (SD) & $1474(513)$ & $1503(505)$ & $1495(533)$ & $1491(517)$ \\
\hline BDI score, mean (SD) & $7.0(1.8)$ & $7.0(1.9)$ & $7.1(1.8)$ & $7.01(1.9)$ \\
\hline Baseline E-RS total score & $10.7(5.6)$ & $10.7(5.8)$ & $10.4(5.7)$ & $10.6(5.7)$ \\
\hline Baseline SGRQ score, mean (SD) & $44.5(16.1)$ & $45.0(16.1)$ & $44.6(16.3)$ & $44.7(16.2)$ \\
\hline Baseline CAT score, mean (SD) & $19.1(5.9)$ & $19.3(6.2)$ & $19.3(6.3)$ & $19.2(6.1)$ \\
\hline Baseline rescue salbutamol, puffs/day, mean (SD) & $2.2(2.6)$ & $2.1(2.3)$ & $2.2(2.5)$ & $2.2(2.5)$ \\
\hline Any cardiac comorbidities ${ }^{\mathrm{e}}, \mathrm{n}(\%)$ & $111(14)$ & $136(17)$ & $117(14)$ & $364(15)$ \\
\hline Any vascular comorbidities ${ }^{f}, \mathrm{n}(\%)$ & $444(55)$ & $434(54)$ & $448(55)$ & $1326(55)$ \\
\hline
\end{tabular}

${ }^{a}$ Includes American Indian/Alaska Native and White, Black/African American and White, Native Hawaiian/Other Pacific Islander and White; ${ }^{b}$ patients could be counted for both LAMA and LABA; ${ }^{C}$ number of exacerbations requiring oral or systemic corticosteroids and/or antibiotics (moderate) in 12 months prior to screening (patients with $>1$ moderate exacerbation or with a severe exacerbation [requiring hospitalisation] were excluded); ${ }^{d}$ an additional 4 ( $\left.<1 \%\right)$ patients with

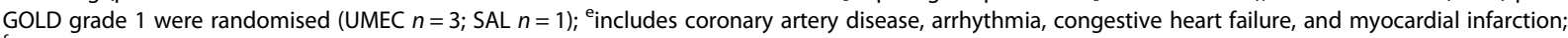
includes hypertension and cerebrovascular accident

$B D I$ Baseline dyspnoea index, CAT COPD Assessment Test, COPD Chronic obstructive pulmonary disease, E-RS Evaluating Respiratory Symptoms, FEV ${ }_{1}$ Forced expiratory volume in $1 \mathrm{~s}, F V C$ Forced vital capacity, GOLD Global Initiative for Chronic Obstructive Lung Disease, $L A B A$ Long-acting $\beta_{2}$-agonist, $L A B D$ Long-acting bronchodilator, LAMA Long-acting muscarinic antagonists, SAL Salmeterol, SD Standard deviation, SGRQ St George's Respiratory Questionnaire, UMEC Umeclidinium, VI Vilanterol 
$\backsim \mathrm{UMEC} N \mathrm{NI} \backsim \mathrm{UMEC} \backsim \mathrm{SAL}$

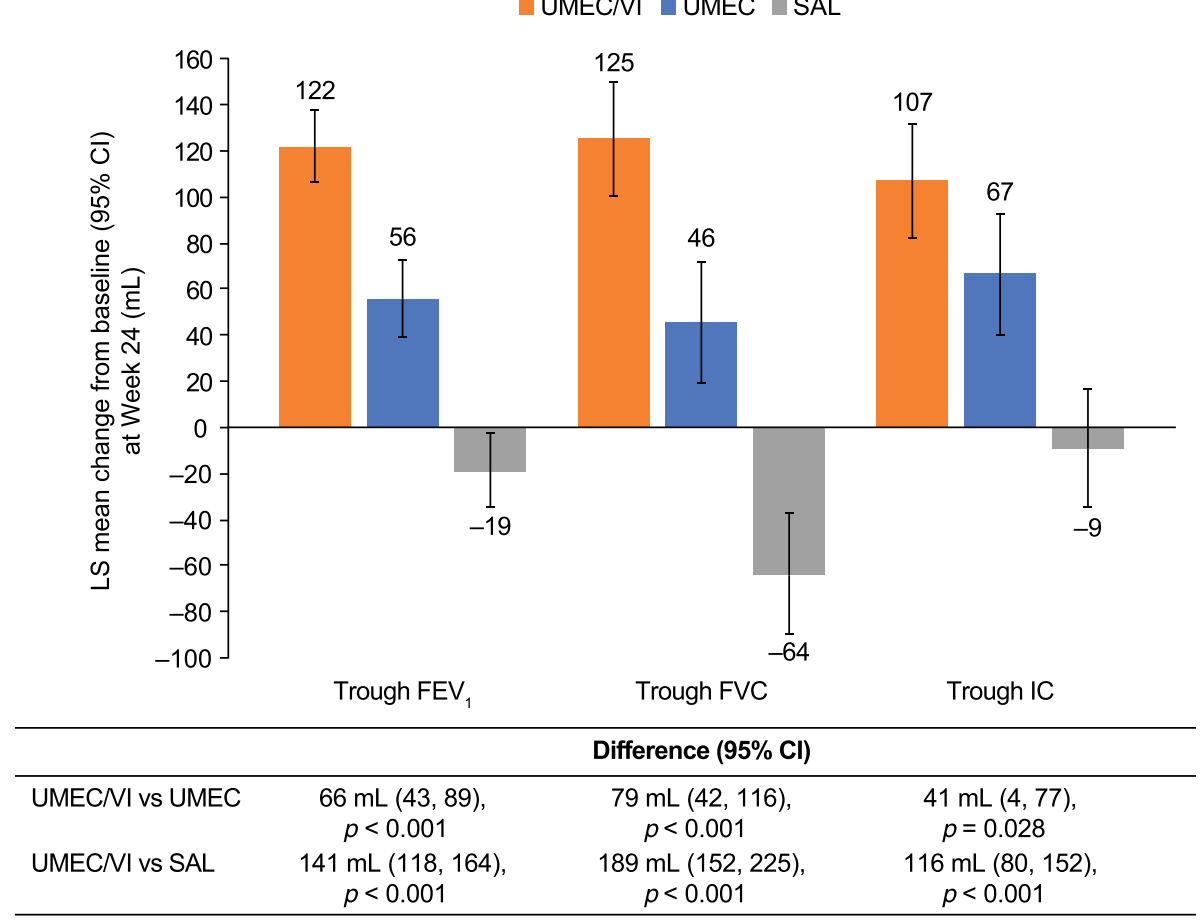

Fig. 2 Lung function outcomes. Cl, confidence interval; $F E V_{1}$, forced expiratory volume in $1 \mathrm{~s}$; FVC, forced vital capacity; IC, inspiratory capacity; $L S$, least squares; SAL, salmeterol; UMEC, umeclidinium; VI, vilanterol

24 was $66 \mathrm{~mL}(95 \% \mathrm{CI}: 43,89)$ and $141 \mathrm{~mL}(95 \% \mathrm{CI}: 118$, 164) greater with UMEC/VI versus UMEC and salmeterol, respectively (both $p<0.001$; Fig. 2). The greater improvements in trough $\mathrm{FEV}_{1}$ observed with UMEC/VI compared with both monotherapies were consistent across all time points (Additional file 8: Figure S3A). For the other spirometric endpoints (trough FVC and trough IC), UMEC/VI demonstrated significantly greater improvements versus both UMEC and salmeterol at all time points analysed (Fig. 2 and Additional file 8: Figures S3B and S3C). Furthermore, for all spirometric endpoints at Week 24, UMEC demonstrated significantly greater improvements versus salmeterol (Additional file 2: Table S2).

Improvements in SAC-TDI score increased over time, with UMEC/VI consistently showing significantly greater improvements versus both monotherapies at all time points (Fig. 3a). All LS mean changes in the three treatment groups exceeded the 1.0 point minimum clinically important difference from baseline in SAC-TDI at Week 24, and responder rates were significantly greater with UMEC/VI versus both UMEC and salmeterol (both $p<0.001$ ) (Table 2 and Additional file 3: Table S3). UMEC/VI demonstrated significantly greater improvements in daily respiratory symptoms measured by E-RS total score compared with both monotherapies at all 4weekly time points analysed (Fig. 3b). The odds of being a responder versus a non-responder in E-RS total score were
1.5 times greater for patients receiving UMEC/VI versus both UMEC and salmeterol (95\% CI: 1.2, 1.9 for each treatment) at Weeks 21-24 (both $p<0.001)$ (Table 2). UMEC/ VI showed statistically significant improvements at Weeks 21-24 for both LS mean change from baseline and proportion of responders across all E-RS subdomains (breathlessness, cough and sputum, and chest scores) compared with UMEC and SAL, except for LS mean change from baseline in E-RS cough and sputum score versus UMEC, which was not significant (Additional file 4: Table S4). UMEC/VI demonstrated significantly greater improvements from baseline in the percentage of rescue medication-free days and significantly fewer mean inhalations per day compared with either monotherapy over Weeks 1-24 according to the e-diaries (Fig. 3c and Table 2). A greater proportion of patients receiving UMEC/VI $(473 / 707 ; 67 \%)$ rated the overall severity of their COPD as improved from baseline to Week 24 compared with 393/638 (62\%) patients receiving UMEC and 413/674 (61\%) patients receiving salmeterol. The ordered odds of improvement versus no improvement were significantly higher for patients receiving UMEC/VI compared with UMEC or salmeterol at all time points (all $p \leq 0.001$ ) (Table 2 and Additional file 3: Table S3).

When considering symptomatic PROs, there were no differences between UMEC and salmeterol for SACTDI, E-RS total score, rescue medication use, or the ordered odds of improvement in patients' global 


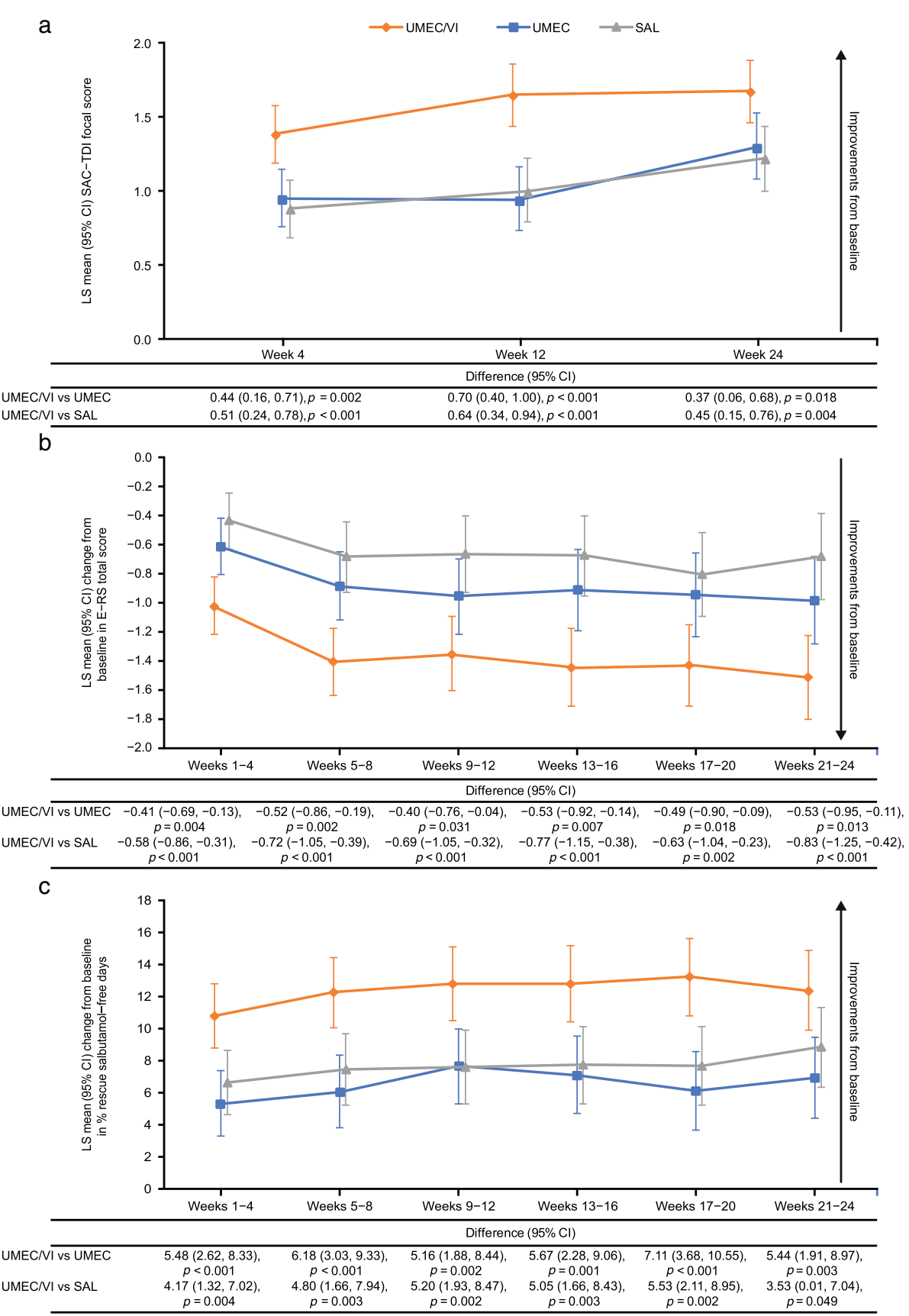

Fig. 3 Symptom severity outcomes (SAC-TDI (a), E-RS (b), \% rescue salbutamol-free days (c)). Cl, confidence interval; COPD, chronic obstructive pulmonary disease; E-RS, Evaluating Respiratory Symptoms-COPD; LS, least squares; SAC-TDI, self-administered computerised Transition Dyspnoea Index; SAL, salmeterol; UMEC, umeclidinium; VI, vilanterol

assessment of disease severity at the final assessment period (Additional file 2: Table S2) or at any other time point.

Statistically significant improvements in the odds of being a responder versus a non-responder in SGRQ were observed with UMEC/VI versus salmeterol at all time points (OR: $1.27-1.49, p<0.05$ ), and at Weeks 4 and 12 versus UMEC (OR: 1.33-1.34, $p<0.01$ ) (Table 2 and
Additional file 3: Table S3). UMEC/VI demonstrated clinically meaningful improvements in LS mean change from baseline in SGRQ total score over Weeks 12-24. Statistically significant improvements with UMEC/VI versus salmeterol were observed at all time points (all $p<0.05$ ); however, statistically significant improvements were not observed versus UMEC (Table 2 and Additional file 9: Figure S4A). UMEC/VI demonstrated significantly greater 
Table 2 LS mean change from baseline and proportion of responders for patient reported-outcomes

\begin{tabular}{|c|c|c|c|}
\hline & $\begin{array}{l}\text { UMECNI } \\
(N=812)\end{array}$ & $\begin{array}{l}\text { UMEC } \\
(N=804)\end{array}$ & $\begin{array}{l}\text { SAL } \\
(N=809)\end{array}$ \\
\hline \multicolumn{4}{|l|}{ Symptom severity outcomes } \\
\hline \multicolumn{4}{|l|}{ SAC-TDI focal score at Week 24} \\
\hline LS mean $(95 \% \mathrm{Cl})$ & $1.68(1.46,1.89)$ & $1.30(1.08,1.53)$ & $1.22(1.00,1.44)$ \\
\hline UMECNI vs comparator mean difference $(95 \% \mathrm{Cl})$ & - & $\begin{array}{l}0.37(0.06,0.68) \\
p=0.018\end{array}$ & $\begin{array}{l}0.45(0.15,0.76) \\
p=0.004\end{array}$ \\
\hline SAC-TDI responders ${ }^{\mathrm{a}}, \mathrm{n} / \mathrm{N}(\%)$ & 403/806 (50) & $332 / 799(42)$ & $330 / 807(41)$ \\
\hline UMECNI vs comparator odds ratio (95\% Cl) & - & $\begin{array}{l}1.43(1.17,1.75) \\
p<0.001\end{array}$ & $\begin{array}{l}1.48(1.21,1.81) \\
p<0.001\end{array}$ \\
\hline \multicolumn{4}{|l|}{ E-RS total score at Weeks 21-24 } \\
\hline LS mean CFB $(95 \% \mathrm{Cl})$ & $-1.52(-1.81,-1.23)$ & $-0.99(-1.29,-0.69)$ & $-0.69(-0.98,-0.39)$ \\
\hline UMECNI vs comparator mean difference $(95 \% \mathrm{Cl})$ & - & $\begin{array}{l}-0.53(-0.95,-0.11) \\
p=0.013\end{array}$ & $\begin{array}{l}-0.83(-1.25,-0.42) \\
p<0.001\end{array}$ \\
\hline E-RS responders ${ }^{b}, n / N(\%)$ & 290/809 (36) & $219 / 800(27)$ & $217 / 808(27)$ \\
\hline UMECNI vs comparator odds ratio $(95 \% \mathrm{Cl})$ & - & $\begin{array}{l}1.52(1.22,1.89) \\
p<0.001\end{array}$ & $\begin{array}{l}1.53(1.23,1.90) \\
p<0.001\end{array}$ \\
\hline \multicolumn{4}{|l|}{ Rescue medication use at Weeks 1-24 } \\
\hline \multicolumn{4}{|l|}{ Mean inhalations/day } \\
\hline LS mean CFB $(95 \% \mathrm{Cl})$ & $-0.61(-0.71,-0.50)$ & $-0.28(-0.38,-0.17)$ & $-0.32(-0.43,-0.22)$ \\
\hline UMECNI vs comparator mean difference $(95 \% \mathrm{Cl})$ & - & $\begin{array}{l}-0.33(-0.48,-0.18) \\
p<0.001\end{array}$ & $\begin{array}{l}-0.28(-0.43,-0.14) \\
p<0.001\end{array}$ \\
\hline \multicolumn{4}{|l|}{$\%$ rescue medication-free days } \\
\hline LS mean CFB $(95 \% \mathrm{Cl})$ & $12.39(10.28,14.50)$ & $6.55(4.42,8.68)$ & $7.68(5.55,9.80)$ \\
\hline UMECNI vs comparator mean difference $(95 \% \mathrm{Cl})$ & - & $\begin{array}{l}5.84(2.84,8.84) \\
p<0.001\end{array}$ & $\begin{array}{l}4.71(1.72,7.70) \\
p=0.002\end{array}$ \\
\hline \multicolumn{4}{|l|}{ Global assessment of disease severity ${ }^{\complement}$ at Week 24} \\
\hline $\begin{array}{l}\text { UMEC } N \text { I vs comparator ordered odds ratio for improvement in } \\
\text { response category }(95 \% \mathrm{Cl})\end{array}$ & - & $\begin{array}{l}1.38(1.14,1.67) \\
p=0.001\end{array}$ & $\begin{array}{l}1.38(1.14,1.68) \\
p<0.001\end{array}$ \\
\hline \multicolumn{4}{|l|}{ Health status outcomes } \\
\hline \multicolumn{4}{|l|}{ SGRQ total score at Week 24} \\
\hline LS mean CFB $(95 \% \mathrm{Cl})$ & $-4.98(-5.89,-4.07)$ & $-5.23(-6.18,-4.28)$ & $-3.29(-4.22,-2.36)$ \\
\hline UMECNI vs comparator mean difference $(95 \% \mathrm{Cl})$ & - & $\begin{array}{l}0.25(-1.07,1.57) \\
p=0.709\end{array}$ & $\begin{array}{l}-1.69(-2.99,-0.39) \\
p=0.011\end{array}$ \\
\hline SGRQ responders ${ }^{\mathrm{d}}, \mathrm{n} / \mathrm{N}(\%)$ & $366 / 811(45)$ & $329 / 802(41)$ & 291/809 (36) \\
\hline UMECNI vs comparator odds ratio (95\% Cl) & - & $\begin{array}{l}1.21(0.99,1.48) \\
p=0.063\end{array}$ & $\begin{array}{l}1.49(1.22,1.83) \\
p<0.001\end{array}$ \\
\hline \multicolumn{4}{|l|}{ CAT score at Week 24} \\
\hline LS mean CFB $(95 \% \mathrm{Cl})$ & $-3.5(-3.9,-3.1)$ & $-3.4(-3.9,-3.0)$ & $-2.9(-3.4,-2.5)$ \\
\hline UMECNI vs comparator mean difference $(95 \% \mathrm{Cl})$ & - & $\begin{array}{l}0.0(-0.6,0.6) \\
p=0.891\end{array}$ & $\begin{array}{l}-0.5(-1.1,0.1) \\
p=0.074\end{array}$ \\
\hline CAT responders ${ }^{\mathrm{e}}, \mathrm{n} / \mathrm{N}(\%)$ & $447 / 812(55)$ & $385 / 804(48)$ & $406 / 809(50)$ \\
\hline UMECNI vs comparator odds ratio $(95 \% \mathrm{Cl})$ & - & $\begin{array}{l}1.35(1.11,1.65) \\
p=0.003\end{array}$ & $\begin{array}{l}1.23(1.01,1.50) \\
p=0.037\end{array}$ \\
\hline
\end{tabular}

${ }^{\mathrm{a} S A C-T D I}$ responders were defined as a $\geq 1$-unit improvement from baseline; ${ }^{\mathrm{b}} \mathrm{E}-\mathrm{RS}$ responders were defined as a reduction of $\geq 2$ from baseline; ${ }^{\mathrm{C}}$ overall assessment of change in COPD severity was rated using a seven-point Likert scale ('Much Better', 'Slightly Better', 'Better', 'No Change', 'Slightly Worse', 'Worse', 'Much Worse'). Ordered response ratios were reported as odds of better response category; ${ }^{d}$ SGRQ responders were defined as a $\geq 4$-point reduction from baseline; ${ }^{\text {CCAT }}$ responders were defined as a $\geq 2$-unit improvement from baseline

CAT COPD Assessment Test, CFB Change from baseline, CI, COPD Chronic obstructive pulmonary disease; e-diary, electronic diary, E-RS Evaluating Respiratory Symptoms-COPD, $L S$ Least squares, $n / N$ number of responders/number of patients with analysable data, SAC-TDI Self-administered computerised Transition Dyspnoea Index, SAL Salmeterol, SGRQ St George's Respiratory Questionnaire, UMEC Umeclidinium, VI Vilanterol

improvements in the proportion of CAT responders versus UMEC at Week $12(p<0.05)$ and versus both UMEC and salmeterol at Week 24 (both $p<0.05$ ) (Table 2 and Additional file 3: Table S3). Similar improvements in LS mean change from baseline CAT total scores over 24 weeks were seen for all treatment groups, with no significant differences from baseline with UMEC/VI versus either monotherapy at any time point, except for versus 
salmeterol at Week 12 (Additional file 9: Figure S4B). When considering UMEC versus salmeterol at Week 24, statistical significance was achieved for SGRQ, which favoured UMEC in terms of both proportion of responders $(p=0.045)$ and LS mean difference $(p=0.004)$; however, no significant difference was observed at other time points, or on CAT score or response rate at any time point (Additional file 2: Table S2).

Overall, the probability of an individual patient experiencing a CID within 24 weeks ranged between 49 and $73 \%$ for all treatments across the different composite definitions, with a consistently lower probability in patients receiving UMEC/VI compared with UMEC and salmeterol (Fig. 4). For all CID definitions, including the $\mathrm{FEV}_{1}$-free definition, fewer CID events were observed with UMEC/VI, demonstrating increased protection from COPD deteriorations compared with both monotherapies (Fig. 5). The probability of having a moderate/ severe exacerbation CID event up to Day 168 was low across all treatments (Additional file 10: Figure S5 and Additional file 11: Figure S6). Overall, 363 patients experienced a moderate $(304 / 363$ [83.7\%]) or severe (59/363 [16.3\%]) exacerbation (UMEC/VI: 101/812 [12\%]; UMEC: 116/804 [14\%]; salmeterol: 146/809 [18\%]). The probability $(95 \% \mathrm{CI})$ of a first moderate or severe exacerbation to Day 168 was $12.8 \%$ (10.7, 15.4), 16.1\% (13.6, 19.0), and 19.4\% $(16.7,22.4)$ for UMEC/VI, UMEC, and salmeterol, respectively. The HR (95\% CI) for risk of a first moderate or severe exacerbation was $0.81(0.62,1.05 ; p=0.114)$ with $\mathrm{UMEC/VI}$ versus UMEC, and $0.64(0.50,0.83 ; p<0.001)$ with UMEC/ VI versus salmeterol. There was no significant difference in the occurrence of severe exacerbations between treatment groups. Patients were less likely to experience a moderate or severe exacerbation compared with the probability of having one of the other CID component events (trough $\mathrm{FEV}_{1}$, SGRQ, CAT, TDI; UMEC/VI [22-36\%], UMEC [31-39\%], and salmeterol 36-43\%]) (Additional file 10: Figure S5 and Additional file 11: Figure S6). UMEC/VI significantly reduced the risk of all individual CID component events versus salmeterol $(p<0.05)$, and the $\mathrm{FEV}_{1}$ and TDI components versus UMEC $(p<0.001)$ (Additional file 10: Figure S5 and Additional file 11: Figure S6).

The probability of experiencing a short-term CID within 24 weeks was lower for UMEC compared with salmeterol for all three CID definitions (Additional file 2: Table S2). For individual component CID events, UMEC significantly reduced the risk of $\mathrm{FEV}_{1}$ events versus salmeterol $(p<0.001)$; however, significant differences were not observed for other individual CID component events (Additional file 2: Table S2).

The overall incidence of on-treatment AEs and SAEs was similar across treatment groups, and the incidence of drug-related AEs was low ( $\leq 5 \%)$ (Table 3). The types of AEs observed were representative of known effects of anticholinergics or $\beta_{2}$-agonists, with the most frequent $\mathrm{AE}$ in all treatment groups being nasopharyngitis

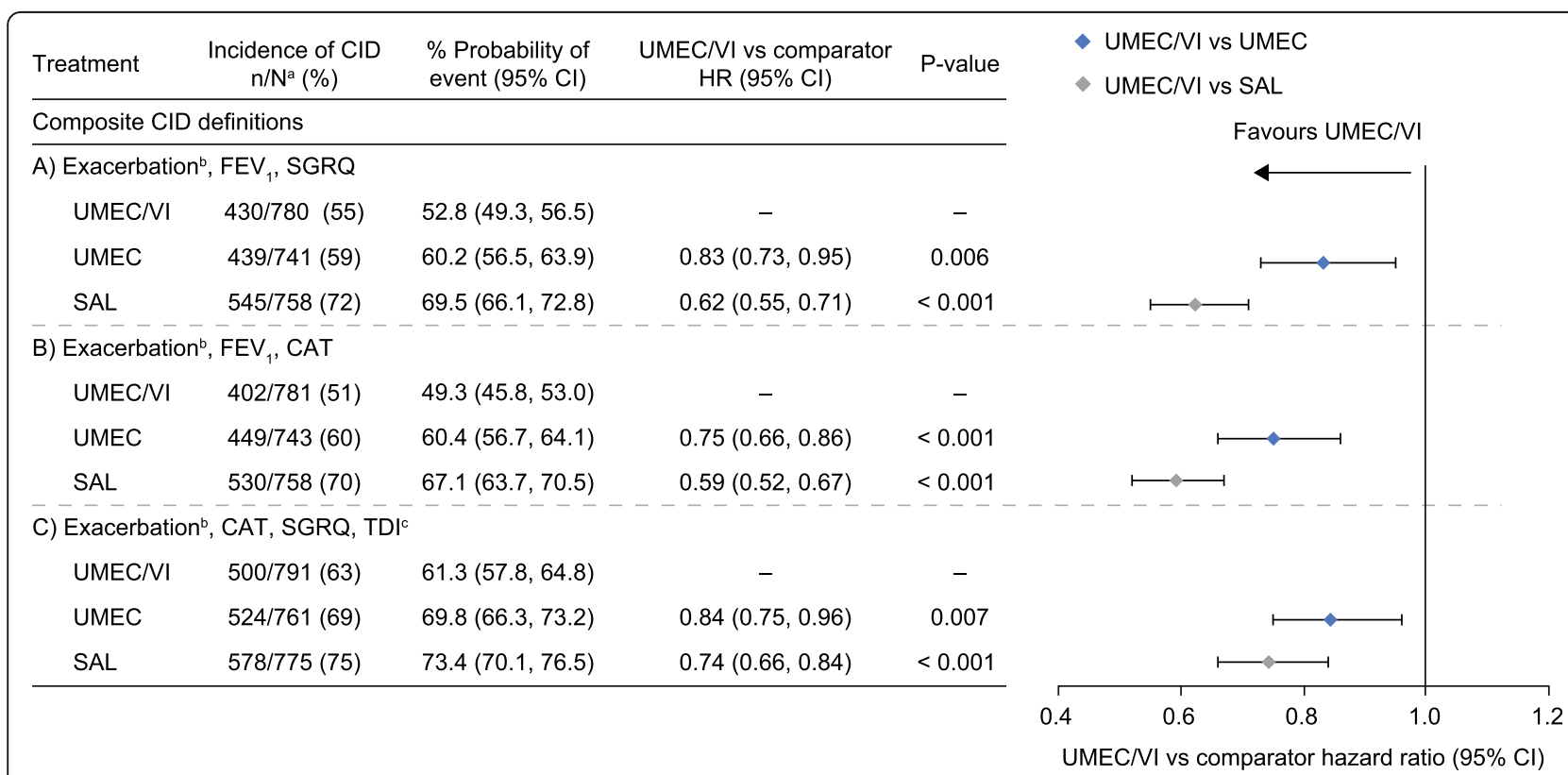

Fig. 4 Risk of a first CID up to Day 168 across multiple composite definitions. a N, patients with at least 1 post baseline assessment (not including exacerbations) for at least one of the individual components or patients who had an exacerbation; $\mathbf{b}$ moderate/severe exacerbation; $\mathbf{c}$ assessed using a self-administered computerised version. CAT, COPD Assessment Test; $C l$, confidence interval; CID, clinically important deterioration; COPD, chronic obstructive pulmonary disease; $F E V_{1}$ trough forced expiratory volume in $1 \mathrm{~s} ; H R$, hazard ratio; $n$, number of patients with an event; TDI, Transition Dyspnoea Index; SAL, salmeterol; SGRQ, St George's Respiratory Questionnaire; UMEC, umeclidinium; VI, vilanterol 
A

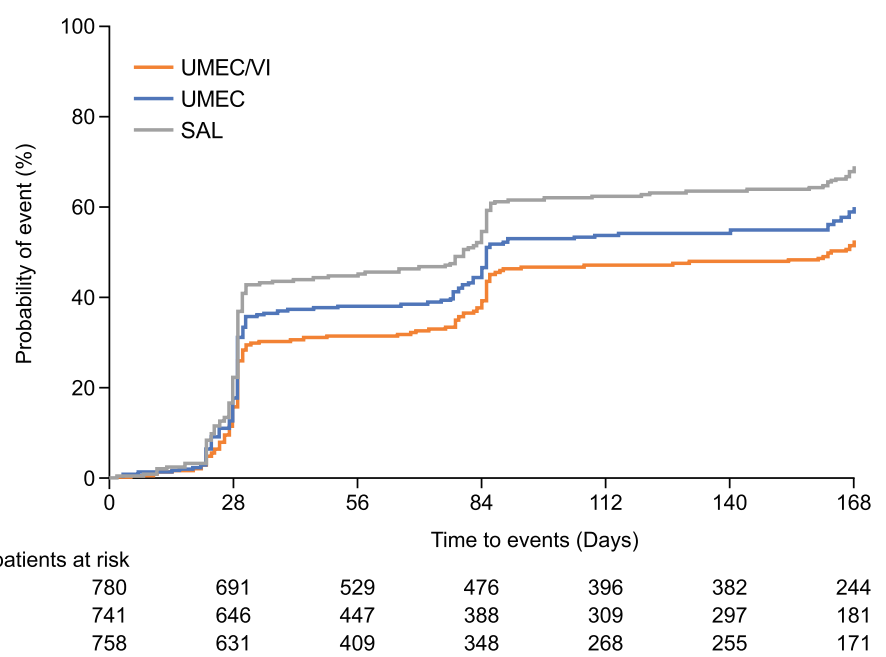

$\begin{array}{lrllllll}\text { Number of patients at risk } & & & & & \\ \text { UMEC/NI } & 780 & 691 & 529 & 476 & 396 & 382 & 244 \\ \text { UMEC } & 741 & 646 & 447 & 388 & 309 & 297 & 181 \\ \text { SAL } & 758 & 631 & 409 & 348 & 268 & 255 & 171\end{array}$

B

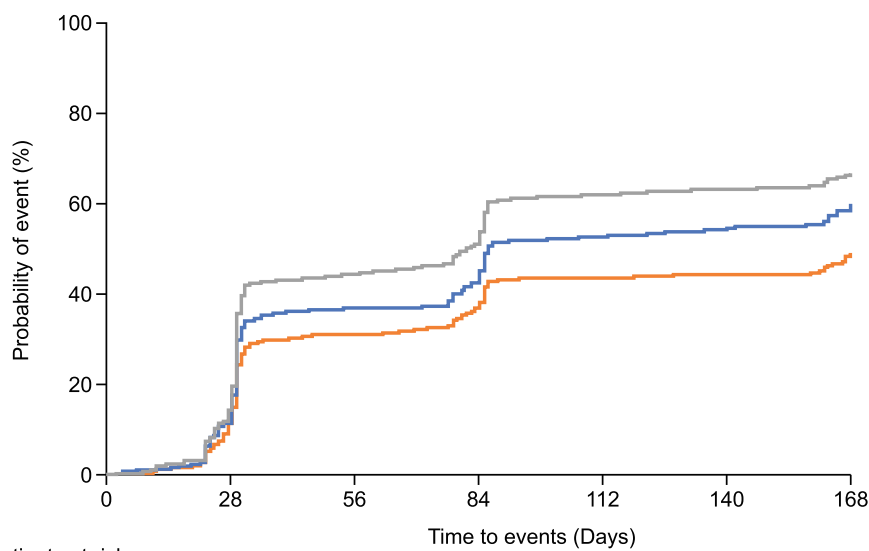

Number of patients at risk

UMEC/VI $\quad 781$

UMEC

$\begin{array}{llllll}693 & 533 & 480 & 421 & 407 & 255 \\ 646 & 460 & 398 & 320 & 304 & 185 \\ 645 & 412 & 357 & 269 & 259 & 162\end{array}$

SAL

743
758

645

412

357

C

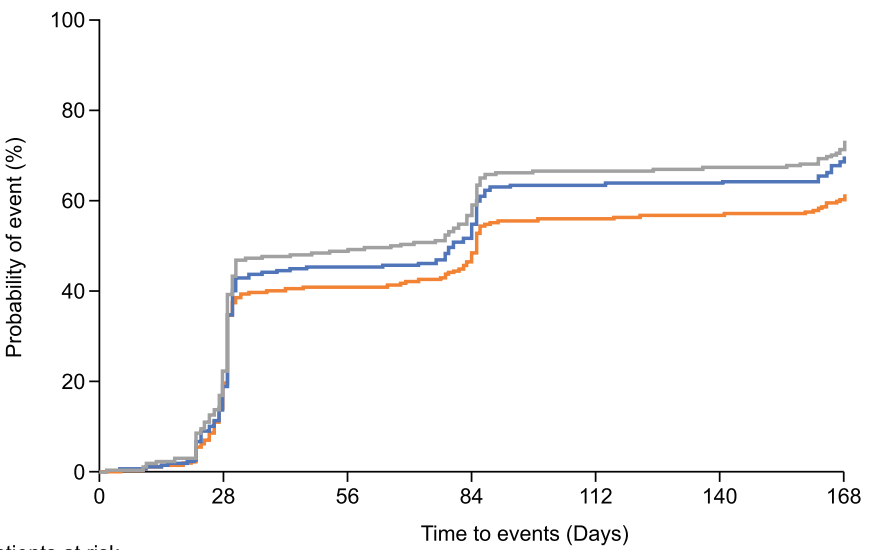

Number of patients at risk

UMEC/VI

risk
791
761

$\begin{array}{ll}680 & 459 \\ 656 & 410\end{array}$

411

332

UMEC

761
775

643

410
391

348

$257-323$

$\begin{array}{ll}257 & 251 \\ 247 & 240\end{array}$

Fig. 5 (See legend on next page.) 
(See figure on previous page.)

Fig. 5 Kaplan-Meier plots of time to first CID for three definitions ${ }^{\mathrm{a}} .{ }^{\mathrm{a}} \mathrm{CID}$ was defined as: a - a first moderate or severe exacerbation, and/or a trough $\mathrm{FEV}_{1}$ decrease from baseline of $\geq 100 \mathrm{~mL}$, and/or a deterioration in SGRQ $\geq 4$ units from baseline; $\mathbf{b}$ - a first moderate or severe exacerbation, and/or a trough $\mathrm{FEV}_{1}$ decrease from baseline of $\geq 100 \mathrm{~mL}$, and/or a deterioration in CAT $\geq 2$ units from baseline; $\mathbf{c}-\mathrm{a}$ first moderate or severe exacerbation, and/or a deterioration in SGRQ $\geq 4$ units from baseline and/or a deterioration in CAT $\geq 2$ units from baseline and/or a TDI deterioration $\geq 1$ unit decrease from baseline. CAT, COPD Assessment Test; CID, clinically important deterioration; FEV , forced expiratory volume in $1 \mathrm{~s}$; SAL, salmeterol; SGRQ, St George's Respiratory Questionnaire; TDI, transition dyspnoea index; UMEC, umeclidinium; VI, vilanterol

$(\leq 11 \%)$. Incidence of non-fatal SAEs was similar across treatment groups $(4-6 \%)$ and none were considered drug-related. A total of eight fatal SAEs were reported, four each in the UMEC/VI (any cardiac disorder: $n=3$, and pneumonia: $n=1$ ) and UMEC (any cardiac disorder: $\mathrm{n}=1$, and any respiratory, thoracic and mediastinal disorder: $\mathrm{n}=3$ ) arms; none were considered drug-related by the investigators. Consistent with previous studies, the incidence of fatal cardiovascular SAEs was $<1 \%$ in all treatment groups.

\section{Discussion}

The EMAX trial is the largest 24-week RCT to date to compare dual- versus mono-bronchodilator therapies in symptomatic patients with COPD who were not receiving ICS either at baseline or concurrently during the study treatment period. It is also the first prospective assessment of the composite CID endpoint as a marker of short-term disease worsening and treatment failure, in patients receiving LAMA/LABA combination therapy compared with LAMA and LABA monotherapies. The study provides consistent evidence that confirms the known incremental benefits of dual bronchodilation on lung function compared with mono-bronchodilator therapy $[2,5,6,8]$ and extends current knowledge by providing a detailed assessment of the symptomatic benefits of dual bronchodilation and its potential to reduce treatment failure in symptomatic COPD.

UMEC/VI demonstrated consistent early, sustained, and similar improvements for all symptomatic outcomes compared with UMEC and salmeterol. These symptom improvements appear not to fully relate to the level of spirometry improvements versus the monotherapies, where $a \geq 2$-fold higher magnitude of spirometry improvement was observed for UMEC/VI versus salmeterol compared with UMEC/VI versus UMEC. In contrast, the level of symptom benefits observed when comparing UMEC/VI versus UMEC and versus salmeterol were broadly comparable. In addition, despite consistent improvements in all spirometry parameters in favour of UMEC versus salmeterol, no symptom benefits were observed between the

Table 3 Adverse events

\begin{tabular}{|c|c|c|c|}
\hline & UMECNI $(N=812)$ & UMEC $(N=804)$ & $\operatorname{SAL}(N=809)$ \\
\hline \multicolumn{4}{|l|}{$A E, n(\%)$} \\
\hline $\mathrm{AE}$ & $315(39)$ & $316(39)$ & $314(39)$ \\
\hline Drug-related $\mathrm{AE}$ & $29(4)$ & $37(5)$ & $27(3)$ \\
\hline AE leading to study withdrawal & $32(4)$ & $36(4)$ & $26(3)$ \\
\hline \multicolumn{4}{|l|}{ SAE, n (\%) } \\
\hline Non-fatal SAE & $46(6)$ & $31(4)$ & $38(5)$ \\
\hline Drug-related non-fatal SAE & 0 & 0 & 0 \\
\hline Fatal SAE & $4(<1)$ & $4(<1)$ & 0 \\
\hline Drug-related fatal SAE & 0 & 0 & 0 \\
\hline \multicolumn{4}{|l|}{ Most frequent $A E s^{\mathrm{a}}, \mathrm{n}(\%)$} \\
\hline Nasopharyngitis & $68(8)$ & $87(11)$ & $84(10)$ \\
\hline Upper respiratory tract infection & $19(2)$ & $12(1)$ & $20(2)$ \\
\hline Influenza & $20(2)$ & $9(1)$ & $18(2)$ \\
\hline Back pain & $10(1)$ & $13(2)$ & $15(2)$ \\
\hline Cough & $14(2)$ & $11(1)$ & $10(1)$ \\
\hline Headache & $10(1)$ & $17(2)$ & $6(<1)$ \\
\hline
\end{tabular}

${ }^{a}$ The incidence of fatal cardiovascular SAEs was $<1 \%$ in all treatment groups, with three cardiac disorders observed in the UMEC/VI arm and one in the UMEC arm

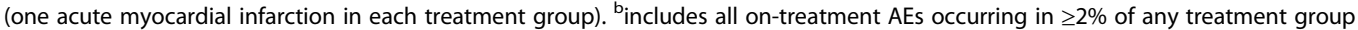

$A E$ Adverse event, SAE Serious adverse event, SAL Salmeterol, UMEC Umeclidinium, VI Vilanterol 
monotherapies at any time point. These findings indicate that the differences in trough spirometry observed between the treatment arms may not capture the inherent daily variability of airway tone and fluctuations in symptoms recalled by patients over longer periods of assessment. Nevertheless, these findings support the current GOLD strategy document, which indicates no overall preference for LAMA or LABA therapy in symptomatic low-risk patients [1].

This study is the first to prospectively assess the CID composite endpoint as a measure of disease stability and freedom from short-term disease worsening/treatment failure in symptomatic patients receiving three different long-acting bronchodilator therapies. Previous studies have retrospectively tested different CID definitions to better understand the heterogeneity of short-term worsening/treatment failure [8,13-16], whereas this study has prospectively assessed three different definitions including one focused exclusively on PROs that does not include a decrease in $\mathrm{FEV}_{1}$. Previous studies have shown that more than half of patients receiving monobronchodilator therapy experience short-term worsening as measured by CID over 24 weeks, a finding confirmed in this trial. In keeping with previous post hoc analyses, this prospective study also shows that the risk of a first CID is reduced by dual-bronchodilator maintenance therapy $[8,13-15,22]$. In this study, UMEC/VI consistently provided increased protection from early treatment failure versus UMEC and salmeterol across all three composite CID definitions examined, including the CID definition that excluded a trough $\mathrm{FEV}_{1}$ decrease. Together, these findings provide additional evidence to support early intensification of bronchodilation in symptomatic patients with COPD, before a high exacerbation risk develops. In addition, this study provides further evidence that the concept of the composite CID endpoint has the potential to monitor individual patients following initiation of standard of care therapy to determine future prognosis. Indeed, short-term CID and lack of disease stability, using $\mathrm{FEV}_{1}$, SGRQ and exacerbations, have been demonstrated to be predictors of sustained long-term deterioration and poor clinical outcomes, including an increase in hospital admissions and mortality over 3-4 years [13, 22-24].

UMEC/VI achieved significant reductions in the risk of moderate or severe exacerbations versus salmeterol, but not versus UMEC. These findings are compatible with a meta-analysis of previous bronchodilator studies, which demonstrated clear benefits in reducing exacerbation risk with dual therapy versus LABAs, yet inconclusive findings for dual therapies versus LAMAs [5]. Likewise, our findings are consistent with results from other studies that evaluated the effect of dual-bronchodilator therapy in reducing exacerbation risk versus LAMA monotherapy in populations at increased exacerbation risk [9, 10]. In contrast to the current study, these trials all allowed continued use of ICS alongside LAMA/LABA or LAMA therapy. Therefore, the absence of a conclusive add-on LABA effect to LAMA bronchodilation in patients at low or high risk of exacerbations cannot be confirmed, either in the absence or presence of ICS. In the absence of large, longterm exacerbation studies of initation of long-acting bronchodilator therapy in patients not receiving ICS, there remains a need to quantify what may be a small benefit in exacerbation protection in these patients and to determine whether it is clinically useful.

All treatments were well-tolerated with no unexpected AEs, a finding in line with previous studies [7, 25, 26]. The greater efficacy of UMEC/VI compared with UMEC and salmeterol was seen with no increase in safety concerns compared with monotherapies. Additionally, more patients on UMEC/VI were able to complete the 24-week study, providing further indication of treatment benefit, including tolerability and protection from deterioration, with UMEC/VI compared with LAMA and LABA monotherapies. It should be noted that fatal SAEs were only observed in treatment arms containing UMEC; however, incidence was $<1 \%$ and consistent with the incidence observed in previous studies [7] and no SAEs were considered to be drug-related by the investigators.

A major strength of this study is that it is the first and largest RCT comparing dual bronchodilation versus monotherapy in symptomatic patients with COPD who were not receiving ICS and it thus fills an important knowledge gap. Patients who are not receiving concurrent ICS therapy may be considered to have early, less severe COPD and may be ideal candidates for early optimisation to dual bronchodilation. Our comprehensive assessment of the clinical impact of dual bronchodilation with a LAMA/LABA indicates consistent additional benefits over monotherapy across a range of clinical and functional outcomes including preventing meaningful treatment failure. Unlike studies in high-risk patients, the role of optimising care in symptomatic patients with low risk of exacerbations has been neglected. Consequently, the timing of intensification of care in such patients to prevent future poor outcomes remains uncertain and many patients on long-acting bronchodilator monotherapy continue to experience significant symptoms [27]. The high baseline CAT scores observed in this analysis are in keeping with those reported in patients who experience frequent exacerbations $[9,28]$. Consequently, it is important for physicians to recognise that patients who may not experience frequent exacerbations still face a significant impact on their health and wellbeing, and that the disease burden in low exacerbation risk patients should not be underestimated. Indeed, the symptomatic burden at baseline in this population, who would be considered as typical GOLD group B patients, has 
been shown to be associated with a higher future risk of hospitalised exacerbations and mortality compared with GOLD group A patients [29, 30]. Our findings suggest that there is a need to consider initiation of bronchodilation with LAMA/LABAs, or early escalation to dual therapy, in this symptomatic patient population.

There are potential limitations to consider in interpreting this study. It was powered for the primary endpoint (trough $\mathrm{FEV}_{1}$ ) and for the secondary endpoint SAC-TDI at Week 24 but was not powered to detect differences in other PROs. The failure to include both monocomponents of UMEC/VI as comparator arms may be perceived as a limitation of this study; however, this was necessitated by local country requirements to generate new data only against currently available LAMA and LABA therapies. Unfortunately, VI is not available as a licensed drug; therefore, SAL was instead selected as a comparator LABA for this study. We note that although SAL provided similar efficacy to UMEC on the majority of PROs, a deterioration in $\mathrm{FEV}_{1}$ was observed at Week 24 in patients receiving SAL. This suggests that SAL may have been less effective at sustaining $24 \mathrm{~h}$ bronchodilation overtime than the baseline LABA or LAMA agents that patients were using at study entry. Furthermore, at the time the EMAX study was designed there were insufficient data available to power the study on each CID component type. Additionally, whilst several composite measures of CID were used in this analysis, including a definition that excluded deterioration in lung function, further research is needed to reach a consensus on how to achieve disease stability in COPD and how best to monitor short-term deterioration/treatment failure in individual patients.

\section{Conclusions}

In symptomatic, low exacerbation risk patients with COPD who were not receiving ICS, once-daily UMEC/ VI provided consistent early and sustained improvements in lung function and symptoms and reduced the probability of short-term COPD worsening compared with both UMEC and salmeterol monotherapies, with no additional safety concerns. Our findings suggest that a sizeable group of symptomatic patients may gain important benefits from earlier use of dual versus mono bronchodilators. Research is now required to help predict the patient type most well-suited for early use of dual therapy.

\section{Supplementary information}

Supplementary information accompanies this paper at https://doi.org/10. 1186/s12931-019-1193-9.

Additional file 1: Table S1. Patient-reported outcomes. ${ }^{\mathrm{a}} \mathrm{SAC}-\mathrm{TD}$ responders were defined as $\mathrm{a} \geq 1$-unit improvement from baseline;

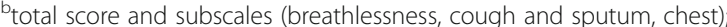
${ }^{C} E-R S$ responders were defined as a reduction of $\geq 2$ from baseline; ${ }^{d} S G R Q$ responders were defined as a $\geq 4$-point reduction from baseline; ${ }^{e} \mathrm{CAT}$ responders were defined as a $\geq 2$-unit improvement from baseline. CAT, COPD Assessment Test; COPD, chronic obstructive pulmonary disease; E-RS, Evaluating Respiratory Symptoms-COPD; LS, least squares; SAC-TDI, self-administered computerised-Transition Dyspnoea Index; SGRQ, St George's Respiratory Questionnaire.

Additional file 2: Table S2. UMEC versus salmeterol comparisons for all outcomes. a'Symptom severity outcomes presented for Week 24 for SACTDI and global assessment of disease severity, for Weeks 21-24 for E-RS, and for Weeks 1-24 for rescue salbutamol use; b data are LS mean (95\% $\mathrm{Cl}$ ); ' $\mathrm{SAC}-\mathrm{TDI}$ responders were defined as a $\geq 1$-unit improvement from baseline; ${ }^{d} E-R S$ responders were defined as a reduction of $\geq 2$ from baseline; ${ }^{~}$ overall assessment of change in COPD severity was rated using a seven-point Likert scale ('Much Better', 'Slightly Better', 'Better', 'No Change', 'Slightly Worse', 'Worse', 'Much Worse'). Ordered response ratios were reported as odds of better response category; ' $S G R Q$ responders were defined as a $\geq 4$-point reduction from baseline; ${ }^{9} \mathrm{CAT}$ responders were defined as a $\geq 2$-unit improvement from baseline. CAT, COPD Assessment Test; Cl, confidence interval; CID, clinically important deterioration; COPD, chronic obstructive pulmonary disease; E-RS, Evaluating Respiratory Symptoms-COPD; FEV 1 , trough forced expiratory volume in $1 \mathrm{sec}$; LS, least squares; n, number of responders/ patients with an event; N, number of patients with analysable data; SAC-TDI, self-administered computerised Transition Dyspnoea Index; SAL, salmeterol; SGRQ, St George's Respiratory Questionnaire; UMEC, umeclidinium; VI, vilanterol.

Additional file 3: Table S3. Proportion of responders for symptom severity and health status outcomes - additional timepoints. ${ }^{a}$ SAC-TDI responders were defined as $a \geq 1$-unit improvement from baseline; ${ }^{b} \mathrm{E}-\mathrm{RS}$ responders were defined as a reduction of $\geq 2$ from baseline; ' overall assessment of change in COPD severity was rated using a seven-point Likert scale ('Much Better', 'Slightly Better', 'Better', 'No Change', 'Slightly Worse', 'Worse', 'Much Worse'). Ordered response ratios were reported as odds of better response category; ${ }^{d} \mathrm{SGRQ}$ responders were defined as $\mathrm{a} \geq 4$-point reduction from baseline; ${ }^{\mathrm{e} C A T}$ responders were defined as $\mathrm{a} \geq 2$-unit improvement from baseline. CAT, COPD Assessment Test; Cl, confidence interval; $C O P D$, chronic obstructive pulmonary disease; ediary, electronic diary; E-RS, Evaluating Respiratory Symptoms-COPD; $\mathrm{n} / \mathrm{N}$, number of responders/number of patients with analysable data; SAC-TDI, self-administered computerised Transition Dyspnoea Index; SAL, salmeterol; SGRQ, St George's Respiratory Questionnaire; UMEC, umeclidinium; VI, vilanterol.

Additional file 4: Table S4. LS mean change from baseline and proportion of responders ${ }^{a}$ for E-RS subdomains at Weeks 21-24. ${ }^{a} \mathrm{E}-\mathrm{RS}$ responders were defined as a reduction of $\geq 1$ unit from baseline for $E-R S$ breathlessness score, and a reduction of $\geq 0.7$ units from baseline for cough and sputum, and chest scores. Cl, confidence interval; CFB, change from baseline; E-RS, Evaluating Respiratory Symptoms-COPD; LS, least squares; $\mathrm{n} / \mathrm{N}$, number of responders/number of patients with analysable data; SAL, salmeterol; UMEC, umeclidinium; VI, vilanterol.

Additional file 5: Table S5. List of investigators.

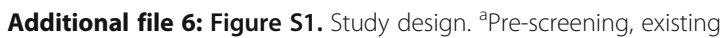
bronchodilator maintenance therapy was limited to a LAMA or LABA, with patients required to be ICS and ICS/LABA free for $\geq 6$ weeks and LAMA/LABA free for $\geq 2$ weeks prior to run-in. ${ }^{b}$ Patients were permitted to continue use of inhaled LAMAs or LABAs and/or study-provided as-needed salbutamol during the run-in period. BID, twice daily; DPI, dry powder inhaler; LABA, long-acting $\beta_{2}$-agonist; LAMA, long-acting muscarinic antagonist; $\mathrm{QD}$, once daily; $\mathrm{R}$, randomisation; $\mathrm{SAL}$, salmeterol; UMEC, umeclidinium; $V$, visit; VI, vilanterol.

Additional file 7: Figure S2. Kaplan-Meier curve time to withdrawal. Note: post hoc analyses showed that 12,19 , and $16 \%$ of patients receiving $U M E C N I, U M E C$, and $S A L$ withdrew from study treatment; UMECNI versus UMEC HR ( $95 \% \mathrm{CI}): 0.60(0.46,0.77), p<0.001$; UMECNI versus SAL HR $(95 \% \mathrm{Cl}): 0.73(0.56,0.96), p=0.022$. SAL, salmeterol; UMEC, umeclidinium; VI, vilanterol. 
Additional file 8: Figure S3. Lung function outcomes (trough $\mathrm{FEV}_{1}[\mathrm{~A}]$, FVC $[B], I C[C]$ ). Cl, confidence interval; $\mathrm{FEV}_{1}$, forced expiratory volume in $1 \mathrm{~S}$; FVC, forced vital capacity; IC, inspiratory capacity; LS, least squares; SAL, salmeterol; UMEC, umeclidinium; VI, vilanterol.

Additional file 9: Figure S4. Health status outcomes (SGRQ score [A], CAT score [B]). CAT, COPD assessment test; $\mathrm{Cl}$, confidence interval; COPD, chronic obstructive pulmonary disease; LS least squares; SGRQ, St George's Respiratory Questionnaire; SAL, salmeterol; UMEC, umeclidinium; Vl, vilanterol.

Additional file 10: Figure S5. Probability of patients experiencing individual CID components to Day 168. CAT, COPD Assessment Test; $\mathrm{Cl}$, confidence interval; $\mathrm{CID}$, clinically important deterioration; $\mathrm{FEV}_{1}$ trough forced expiratory volume in $1 \mathrm{~s}$; HR, hazard ratio; $\mathrm{n} / \mathrm{N}$, number of patients with an event/number of patients with analysable data; TDI, Transition Dyspnoea Index; SAL, salmeterol; SGRQ, St George's Respiratory Questionnaire; UMEC, umeclidinium; VI, vilanterol.

Additional file 11: Figure S6. Kaplan-Meier plots of time to first CID event ${ }^{\mathrm{a}}$. ${ }^{\mathrm{P}}$ Panel $\mathrm{A}$ : first on-treatment moderate/severe exacerbation; Panel B: first decrease from baseline of $\geq 100 \mathrm{~mL}$ in trough $\mathrm{FEV}_{1}$; Panel C: first increase from baseline of $\geq 4$ units in SGRQ total score; Panel D: first increase from baseline of $\geq 2$ units in CAT score; Panel E: first decrease of $\geq 1$ unit in SAC-TDI. CAT, COPD Assessment Test; CID, clinically important deterioration; $\mathrm{FEV}_{1}$, forced expiratory volume in $1 \mathrm{~s}$; SAL, salmeterol; SGRQ, St George's Respiratory Questionnaire; TDI, transition dyspnoea index; UMEC, umeclidinium; VI, vilanterol.

\section{Abbreviations}

AEs: Adverse events; BDI: Baseline dyspnoea index; CAT: COPD Assessment Test; CID: Clinically important deterioration; Cls: Confidence intervals; COPD: Chronic Obstructive Pulmonary Disease; E-diary: Electronic diary; EMAX: Early MAXimisation of bronchodilation for improving COPD stability; E-RS: Evaluating Respiratory Symptoms; $F_{E V}$ : Forced expiratory volume in $1 \mathrm{~s}$; FVC: Forced vital capacity; GOLD: Global Initiative for Chronic Obstructive Lung Disease; HR: Hazard ratios; IC: Inspiratory capacity; ICS: Inhaled corticosteroids; ITT: Intent-to-treat; LABAs: Long-acting $\beta_{2}$-agonists; LAMAs: Long-acting muscarinic antagonists; LS: Least squares; OR: Odds ratios; PROs: Patient-reported outcomes; RCTs: Randomised controlled trials; SAC-TDI: Self-administered computerised-Transition Dyspnoea Index; SAEs: Serious adverse events; SAL: Salmeterol; SD: Standard deviation; SGRQ: St George's Respiratory Questionnaire; UMEC: Umeclidinium; UMEC/ VI: Umeclidinium/vilanterol

\section{Acknowledgements}

The authors would like to thank the investigators (Additional file 5: Table S5) and their patients who participated in the study, as well as Dalal Mouneimne, GSK, and Maria St. Clare, GSK, for their assistance in this study. Editorial support (in the form of writing assistance during development of the initial draft, assembling tables and figures, collating authors comments, grammatical editing, and referencing) was provided by Meghan Betts of Fishawack Indicia Ltd., UK, and was funded by GSK.

\section{Authors' contributions}

All named authors meet the International Committee of Medical Journal Editors (ICMJE) criteria for authorship for this manuscript, take responsibility for the integrity of the work as a whole, contributed to the writing and reviewing of the manuscript, and have given final approval of the version to be published. All authors had full access to all of the data in this study and take complete responsibility for the integrity of the data and accuracy of the data analysis. FM, LB, and EMK were involved in the acquisition of data and data analysis and interpretation. MLW, LT, IPN, DAL, CC, and PJ were involved in the conception and design of the study and data analysis and interpretation. CFV and IHB were involved in the data analysis and interpretation. MV-B was involved in the conception and design of the study.

\section{Funding}

This study was funded by GlaxoSmithKline (GSK study number: 201749 [NCT03034915]). GSK-affiliated authors had a role in study design, data analysis, data interpretation, and writing of the report and GSK funded the article processing charges and open access fee.

\section{Availability of data and materials}

Anonymised individual participant data and study documents can be requested for further research from www.clinicalstudydatarequest.com.

\section{Ethics approval and consent to participate}

This study was performed according to the Declaration of Helsinki and received appropriate ethical approval. All patients provided written informed consent via a form signed at either the Pre-screening or Screening visit.

\section{Consent for publication}

Not applicable.

\section{Competing interests}

$I H B, D A L, C C, M V-B$, and PWJ are employees of GSK and hold stocks and shares in GSK. IPN and MLW were employees of GSK at the time of the study. LT is a contingent worker on assignment at GSK. FM has received research grants for participating in multicentre trials for AstraZeneca, Boehringer Ingelheim, GSK, Sanofi, and Novartis, and has received unrestricted research grants and personal fees from Boehringer Ingelheim, Grifols, and Novartis. LB has received honoraria for giving a lecture or attending an advisory board for Airsonett, ALK-Abello, AstraZeneca, Boehringer Ingelheim, Chiesi, GSK, Meda, Novartis and Teva. EMK has served on advisory boards, speaker panels or received travel reimbursement from for Amphastar, AstraZeneca, Boehringer Ingelheim, GSK, Mylan, Novartis, Pearl, Sunovion, Teva, and Theravance and has received consulting fees from Cipia and GSK. CFV has received grants from AstraZeneca, Boehringer Ingelheim, GSK, Grifols, Novartis, Bayer-Schering, MSD, and Pfizer, and has received personal fees from AstraZeneca, Boehringer Ingelheim, Chiesi, Cipla, CSL Behring, GSK, Grifols, Menarini, Mundipharma, Novartis, and Teva. ELLIPTA and DISKUS are owned by/licensed to the GSK group of companies.

\section{Author details}

${ }^{1}$ Centre de Pneumologie, Institut universitaire de cardiologie et de pneumologie de Québec, Université Laval, Québec, Canada. ${ }^{2}$ Respiratory Medicine and Allergology, Lund University, Lund, Sweden. ${ }^{3}$ Clinical Research Institute of Southern Oregon, Medford, OR, USA. ${ }^{4}$ Global Specialty \& Primary Care, GSK, Brentford, Middlesex, UK. ${ }^{5}$ Respiratory Research and Development, GSK, Research Triangle Park, NC, USA. ${ }^{6}$ Precise Approach Ltd, contingent worker on assignment at GSK, Stockley Park West, Uxbridge, Middlesex, UK. ${ }^{7}$ Respiratory Research and Development, GSK, Collegeville, PA, USA. ${ }^{8}$ Perelman School of Medicine, University of Pennsylvania, Philadelphia, PA, USA. ${ }^{9}$ Respiratory Discovery Medicine, Respiratory Research and Development, GSK, Stevenage, Hertfordshire, UK. ${ }^{10}$ Department of Medicine, Pulmonary and Critical Care Medicine, University Medical Center Giessen and Marburg, Philipps-Universität Marburg, Germany, Member of the German Center for Lung Research (DZL), Marburg, Germany.

\section{Received: 26 June 2019 Accepted: 20 September 2019}

\section{Published online: 30 October 2019}

\section{References}

1. Global Initiative for Chronic Obstructive Lung Disease (GOLD). Global strategy for the diagnosis, management and prevention of chronic obstructive pulmonary disease. 2019. https://goldcopd.org/wp-content/ uploads/2018/11/GOLD-2019-v1.7-FINAL-14Nov2018-WMS.pdf. Accessed 24 June 2019.

2. Bateman ED, Ferguson GT, Barnes N, Gallagher N, Green Y, Henley M, Banerji D. Dual bronchodilation with QVA149 versus single bronchodilator therapy: the SHINE study. Eur Respir J. 2013;42:1484-94.

3. Calzetta L, Rogliani P, Matera MG, Cazzola M. A systematic review with meta-analysis of dual Bronchodilation with LAMA/LABA for the treatment of stable COPD. Chest. 2016;149:1181-96.

4. Donohue JF, Jones PW, Bartels C, Marvel J, D'Andrea P, Banerji D, Morris DG, Patalano F, Fogel R. Correlations between FEV1 and patientreported outcomes: a pooled analysis of 23 clinical trials in patients with chronic obstructive pulmonary disease. Pulm Pharmacol Ther. 2018:49:11-9

5. Oba Y, Sarva ST, Dias S. Efficacy and safety of long-acting beta-agonist/longacting muscarinic antagonist combinations in COPD: a network metaanalysis. Thorax. 2016;71:15-25. 
6. Donohue JF, Singh D, Munzu C, Kilbride S, Church A. Magnitude of umeclidinium/vilanterol lung function effect depends on monotherapy responses: results from two randomised controlled trials. Respir Med. 2016; 112:65-74.

7. Donohue JF, Maleki-Yazdi MR, Kilbride S, Mehta R, Kalberg C, Church A. Efficacy and safety of once-daily umeclidinium/vilanterol 62.5/25 mcg in COPD. Respir Med. 2013;107:1538-46.

8. Maleki-Yazdi MR, Singh D, Anzueto A, Tombs L, Fahy WA, Naya I. Assessing short-term deterioration in maintenance-naive patients with COPD receiving Umeclidinium/Vilanterol and Tiotropium: a pooled analysis of three randomized trials. Adv Ther. 2017:33:2188-99.

9. Calverley PMA, Anzueto AR, Carter K, Grönke L, Hallmann C, Jenkins C, Wedzicha J, Rabe KF. Tiotropium and olodaterol in the prevention of chronic obstructive pulmonary disease exacerbations (DYNAGITO): a double-blind, randomised, parallel-group, active-controlled trial. Lancet Respir Med. 2018;6:337-44.

10. Wedzicha JA, Decramer M, Ficker JH, Niewoehner DE, Sandström T, Fowler Taylor A, D'Andrea P, Arrasate C, Chen H, Banerji D. Analysis of chronic obstructive pulmonary disease exacerbations with the dual bronchodilator QVA149 compared with glycopyrronium and tiotropium (SPARK): a randomised, double-blind, parallel-group study. Lancet Respir Med. 2013;1: 199-209.

11. Naya I, Tombs L, Lipson DA, Boucot I, Compton C. Impact of prior and concurrent medication on exacerbation risk with long-acting bronchodilators in chronic obstructive pulmonary disease: a post hoc analysis. Respir Res. 2019;20:60.

12. Sion KYJ, Huisman EL, Punekar YS, Naya I, Ismaila AS. A network metaanalysis of long-acting muscarinic antagonist (LAMA) and long-acting $\beta 2-$ agonist (LABA) combinations in COPD. Pulm Ther. 2017;3:297-316.

13. Anzueto AR, Kostikas K, Mezzi K, Shen S, Larbig M, Patalano F, Fogel R, Banerji D, Wedzicha JA. Indacaterol/glycopyrronium versus salmeterol/ fluticasone in the prevention of clinically important deterioration in COPD: results from the FLAME study. Respir Res. 2018;19:121.

14. Anzueto AR, Vogelmeier CF, Kostikas K, Mezzi K, Fucile S, Bader G, Shen S, Banerji D, Fogel R. The effect of indacaterol/glycopyrronium versus tiotropium or salmeterol/fluticasone on the prevention of clinically important deterioration in COPD. Int J Chron Obstruct Pulmon Dis. 2017;12: 1325-37.

15. Naya IP, Tombs L, Lipson DA, Compton C. Preventing clinically important deterioration of COPD with addition of Umeclidinium to inhaled corticosteroid/long-acting $\beta$ (2)-agonist therapy: an integrated post hoc analysis. Adv Ther. 2018;35:1626-38

16. Singh D, Maleki-Yazdi MR, Tombs L, lqbal A, Fahy WA, Naya I. Prevention of clinically important deteriorations in COPD with umeclidinium/vilanterol. Int J Chron Obstruct Pulmon Dis. 2016;11:1413-24.

17. Feldman G, Maltais F, Khindri S, Vahdati-Bolouri M, Church A, Fahy WA, Trivedi R. A randomized, blinded study to evaluate the efficacy and safety of umeclidinium $62.5 \mathrm{mcg}$ compared with tiotropium $18 \mathrm{mcg}$ in patients with COPD. Int J Chron Obstruct Pulmon Dis. 2016;11:719-30.

18. Mahler DA, Witek TJ Jr. The MCID of the transition dyspnea index is a total score of one unit. COPD. 2005:2:99-103.

19. Leidy NK, Murray LT, Monz BU, Nelsen L, Goldman M, Jones PW, Dansie EJ Sethi S. Measuring respiratory symptoms of COPD: performance of the EXACT- respiratory symptoms tool (E-RS) in three clinical trials. Respir Res. 2014:15:124

20. Jones PW. Interpreting thresholds for a clinically significant change in health status in asthma and COPD. Eur Respir J. 2002;19:398-404.

21. Kon SSC, Canavan JL, Jones SE, Nolan CM, Clark AL, Dickson MJ, Haselden BM, Polkey MI, Man WD-C. Minimum clinically important difference for the COPD assessment test: a prospective analysis. Lancet Respir Med. 2014;2: 195-203.

22. Naya I, Compton C, Ismaila AS, Birk R, Brealey N, Tabberer M, Zhu C-Q, Lipson DA, Criner G. Preventing clinically important deterioration with single-inhaler triple therapy in COPD. ERJ Open Res. 2018. https://doi.org/10. 1183/23120541.00047-2018.

23. Naya IP, Tombs L, Muellerova H, Compton C, Jones PW. Long-term outcomes following first short-term clinically important deterioration in COPD. Respir Res. 2018;19:222.

24. Han MK, Halpin DM, Martinez FJ, Miravitlles M, Singh D, de la Hoz A, Voss F, Rabe KF. A composite endpoint of clinically important deterioration in chronic obstructive pulmonary disease and its association with increased mortality: a post hoc analysis of the UPLIFT study. Am J Respir Crit Care. 2018;197:A4245.

25. Celli B, Crater G, Kilbride S, Mehta R, Tabberer M, Kalberg CJ, Church A. Once-daily umeclidinium/vilanterol 125/25 mcg in COPD: a randomized, controlled study. Chest. 2014;145:981-91.

26. Decramer M, Anzueto A, Kerwin E, Kaelin T, Richard N, Crater G, Tabberer M, Harris S, Church A. Efficacy and safety of umeclidinium plus vilanterol versus tiotropium, vilanterol, or umeclidinium monotherapies over 24 weeks in patients with chronic obstructive pulmonary disease: results from two multicentre, blinded, randomised controlled trials. Lancet Respir Med. 2014; 2:472-86.

27. Dransfield MT, Bailey W, Crater G, Emmett A, O'Dell DM, Yawn B. Disease severity and symptoms among patients receiving monotherapy for COPD. Prim Care Respir J. 2011;20:46-53.

28. Mackay AJ, Donaldson GC, Patel AR, Jones PW, Hurst JR, Wedzicha JA. Usefulness of the chronic obstructive pulmonary disease assessment test to evaluate severity of COPD exacerbations. Am J Respir Crit Care Med. 2012; 185:1218-24.

29. Agusti A, Edwards LD, Celli B, MacNee W, Calverley PMA, Müllerova H, Lomas DA, Wouters E, Bakke P, Rennard S, Crim C, Miller BE, Coxson HO, Yates JC, Tal-Singer R. Vestbo J on behalf of the ECLIPSE investigators. Characteristics, stability and outcomes of the 2011 GOLD COPD groups in the ECLIPSE cohort. Eur Respir J. 2013;42:636-46.

30. Gedebjerg A, Szépligeti SK, Wackerhausen LMH, Horváth-Puhó E, Dahl R, Hansen JG, Sørensen HT, Nørgaard M, Lange P, Thomsen RW. Prediction of mortality in patients with chronic obstructive pulmonary disease with the new global initiative for chronic obstructive lung disease 2017 classification: a cohort study. Lancet Respir Med. 2018;6:204-12.

\section{Publisher's Note}

Springer Nature remains neutral with regard to jurisdictional claims in published maps and institutional affiliations.

\section{Ready to submit your research? Choose BMC and benefit from:}

- fast, convenient online submission

- thorough peer review by experienced researchers in your field

- rapid publication on acceptance

- support for research data, including large and complex data types

- gold Open Access which fosters wider collaboration and increased citations

- maximum visibility for your research: over $100 \mathrm{M}$ website views per year

At BMC, research is always in progress.

Learn more biomedcentral.com/submissions 